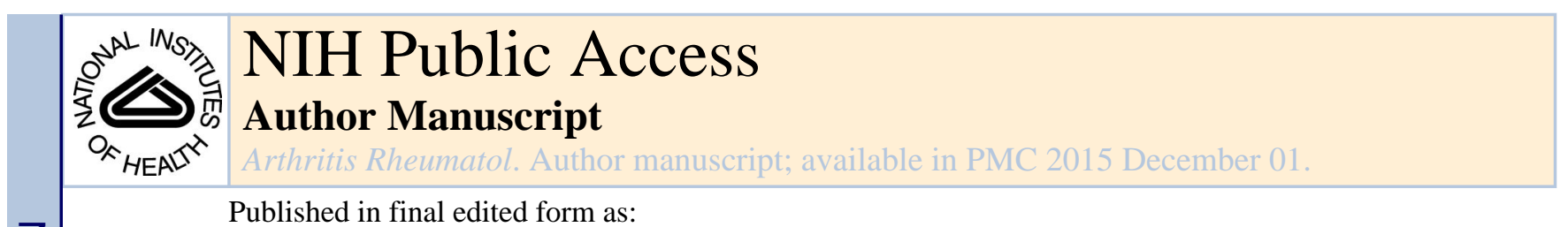

Published in final edited form as:

Arthritis Rheumatol. 2014 December ; 66(12): 3359-3370. doi:10.1002/art.38888.

\title{
Vimentin is a dominant target of in situ humoral immunity in human lupus tubulointerstitial nephritis
}

\author{
Andrew J Kinloch, PhD, Anthony Chang, MD, Kichul Ko, MD, Carole J Henry Dunand, PhD, \\ Scott Henderson, BS, Mark Maienschein-Cleine, PhD, Natalya Kaverina, MD PhD, Brad \\ Rovin, MD, Marlene Salgado Ferrer, BS, Don Wolfgeher, BS, Vladimir Liarski, MD, D James \\ Haddon, PhD, Paul J Utz, MD, Patrick C Wilson, PhD, and Marcus R Clark, MD*
}

\begin{abstract}
Objective-In lupus nephritis (LuN), severe tubulointerstitial inflammation (TII) predicts progression to renal failure. Severe TII is associated with tertiary lymphoid neogenesis and in situ antigen-driven clonal B cell selection. The autoantigen(s) driving in situ B-cell selection in TII are not known. This study aimed to identify the dominant driving autoantigen(s).
\end{abstract}

Methods-Single $\mathrm{CD} 38^{+}$or $\mathrm{Ki}-67^{+} \mathrm{B}$ cells were laser captured from seven $\mathrm{LuN}$ diagnostic biopsies. Eighteen clonally expanded immunoglobulin heavy and light chain variable region pairs were cloned and expressed as monoclonal antibodies. Seven more antibodies were cloned from flow sorted $\mathrm{CD}_{3}{ }^{+}$cells from an eighth biopsy. Antigen characterization was performed using a combination of confocal microscopy, ELISA, screening protoarrays, immunoprecipitation and mass spectrometry. Serum IgG titers to the dominant antigen were determined in $48 \mathrm{LuN}$ and 35 non-nephritic lupus samples using purified antigen-coated arrays. Autoantigen expression was localized by immunohistochemistry and immunofluorescence on normal and LuN kidney.

Results-Eleven of 25 antibodies reacted with cytoplasmic structures, four reacted with nuclei, and none with dsDNA. Vimentin was the only autoantigen identified by both mass spectrometry and by protoarray. Ten of the 11 anti-cytoplasmic TII antibodies directly bound vimentin. Vimentin was highly expressed by tubulointerstitial inflammatory cells, and tested TII antibodies preferentially bound inflamed tubulointerstitium. Finally, high-titers of serum anti-vimentin antibodies were associated with severe TII $(p=0.0001)$.

Conclusion-Vimentin, an antigenic feature of inflammation, is a dominant autoantigen targeted in situ in LuN TII. This adaptive autoimmune response likely feeds forward to worsen TII and renal damage.

\section{Introduction}

Systemic Lupus Erythematosus (SLE) is the archetypical systemic autoimmune disease in which a break in both $\mathrm{B}$ and $\mathrm{T}$ cell tolerance enables pathogenic adaptive immunity to

"Corresponding author: Marcus R Clark, M.D., Section of Rheumatology, Department of Medicine and Gwen Knapp Center for Lupus \& Immunology Research, University of Chicago, JFK R310, 924 E. 57th Street, Chicago, Illinois 60637, Tel.773-702-0202, Fax.

773-702-8702,mclark@medicine.bsd.uchicago.edu.

Other relevant financial disclosures:

AJK and MRC are applicants of a patent pertaining to the use of AVAs in diagnosis of TII. 
ubiquitous nuclear self-antigens[1]. In this systemic model, antibodies and lymphocytes disseminate from secondary lymphoid organs (SLOs) to cause damage in end organs including the kidneys, lungs, skin, gastrointestinal track, brain and heart [2]. Renal inflammation is a common [3,4], severe manifestation of SLE that is often resistant to treatment with cytotoxic therapies [5]. Up to 50\% of SLE patients develop nephritis and up to $50 \%$ of those affected progress to renal failure within five years $[6,7]$.

The principal lesion within the kidney associated with systemic autoimmunity is glomerulonephritis (GN). GN is associated with serum anti-dsDNA antibodies that often deposit in glomeruli $[8,9]$. In animal models, some anti-dsDNA antibodies can induce GN $[10,11]$.

In human lupus nephritis (LuN) tubulointerstitial inflammation (TII) is also common. On renal biopsy, severity of TII, rather than severity of GN, predicts progression to renal failure $[6,7,12]$. Furthermore, unlike GN, severe TII is associated with in situ adaptive immunity. Tertiary lymphoid organ (TLO)-like structures are common in severe TII, including T:B aggregates, plasmablast foci and GCs [13]. In situ antigen-driven selection of B cells occurs in each of these structures. Therefore, human LuN appears to arise from both systemic and in situ autoimmune responses, with the latter more closely associated with a poor prognosis $[6,7,12]$.

The antigens driving in situ adaptive immunity in LuN are not known. Therefore, we characterized a panel of in situ selected IgGs from renal biopsies. Vimentin, an antigen induced in TII, was the most commonly targeted autoantigen. Furthermore, high serum titers of anti-vimentin antibodies (AVAs) were restricted to patients with severe TII. These findings suggest that AVAs might be a useful biomarker of an in situ adaptive immune mechanism associated with severe TII.

\section{Materials and Methods}

\section{Patient samples}

Patients meeting revised 1982 ACR criteria for Systemic Lupus Erythematosus at the University of Chicago and Ohio State University were retrospectively selected. All patients provided informed consent and the study was approved by relevant institutional review boards.

\section{Monoclonal antibody generation}

Briefly, frozen biopsies [13] were sectioned (7 $\mu \mathrm{m})$, adhered to microscope slides, fixed in acetone $\left(-20^{\circ} \mathrm{C}, 10 \mathrm{~min}\right)$, washed with ice cold PBS, and blocked with $10 \%$ donkey serum (DS, Jackson ImmunoResearch). Sections were stained with anti-CD38 (DAKO, $2 \mu \mathrm{g} / \mathrm{ml}$ ) or anti-Ki-67 (Thermo Scientific, $2 \mu \mathrm{g} / \mathrm{ml}$ ) antibodies conjugated with FITC (Life Technologies) in PBS/5\% DS. Positively stained single cells were captured using the Arcturus Pixcell II (Molecular Devices) and Capsure HS LCM caps (Molecular Devices) with an infrared laser $(810 \mu \mathrm{m})$ spot diameter of $7.5 \mu \mathrm{m}, 70 \mathrm{~mW}$ pulse power, $5 \mathrm{~ms}$ pulse duration and $170 \mathrm{~mW}$ voltage [13]. Caps were extracted as described previously [13]. 
One biopsy was digested at $37^{\circ} \mathrm{C}$ for $30 \mathrm{~min}$ in $5 \mathrm{ml}$ digestion buffer $(2 \mu \mathrm{g} / \mathrm{ml}$ collagenase B, $0.2 \mu \mathrm{g} / \mathrm{ml}$ DNaseI, $1 \%$ BSA, $25 \mathrm{mM} \mathrm{NaHCO} 3,10 \mathrm{mM}$ HEPES in HBSS), passed through a $200 \mu \mathrm{m}$ nylon cell strainer and resuspended in FACS buffer. Single CD $19^{+} \mathrm{CD} 38^{+}$cells were sorted into 96 well plates containing lysis buffer. mRNA was reverse transcribed [15] and $\mathrm{V}_{\mathrm{H}}(\mathrm{IgG})$ and $\mathrm{V}_{\mathrm{L}}(\kappa$ and $\lambda$ ) regions PCR amplified, and cloned into TOPO vectors (Life Technologies), sequenced and analyzed $[13,16]$. Paired $\mathrm{V}_{\mathrm{H}}$ and $\mathrm{V}_{\mathrm{L}}$ sequences were subcloned into their respective $\mathrm{IgG} 1$ heavy, $\lambda$, or $\kappa$ expression vectors and human $\mathrm{IgG} 1$ monoclonal antibodies (mAbs) produced [15].

\section{Confocal Microscopy}

For Figure 1, ANA staining patterns of TIN mAbs were visualized using an IIF kit (INOVA diagnostics). For subsequent studies, HEp-2 cells were fixed in methanol [17] and incubated with $\mathrm{mAbs}(50 \mu \mathrm{g} / \mathrm{ml})$, followed by Alexa Fluor 488-conjugated goat anti-human IgG (2 $\mu \mathrm{g} / \mathrm{ml}$ ) and Hoechst 33342 (both Life Technologies). Selected slides were sequentially stained with anti-vimentin V9 $(2 \mu \mathrm{g} / \mathrm{ml}$, DAKO) or anti-giantin $(1 \mu \mathrm{g} / \mathrm{ml}$, Abcam) followed by Alexa Fluor 568-conjugated donkey anti-mouse antibodies ( $2 \mu \mathrm{g} / \mathrm{ml}$, Molecular Probes). Samples were imaged using a Leica SP5 II STED-CW laser scanning confocal microscope.

\section{Protein purification and identification}

Cells were lysed on ice in RIPA buffer $\left(5 \times 10^{6}\right.$ cells $\left./ 0.5 \mathrm{ml}\right)$. Clarified lysates were precleared with protein $\mathrm{G}$ agarose (Pierce) and then incubated with $\mathrm{mAb}$ mixtures (200 $\mu \mathrm{g}$ total pre-bound to protein $\mathrm{G}$ agarose) overnight at $4^{\circ} \mathrm{C}$. Following washing in ice cold IP wash buffer ( $25 \mathrm{mM}$ Tris, $150 \mathrm{mM} \mathrm{NaCl}, \mathrm{pH}$ 7.2), immunoprecipitations were resolved by reducing SDS-PAGE (4-12\% Bis-Tris, Life Technologies). Gels were stained with Instant Blue (Expedeon) and gel tranches (T1-4) excised. Tranches were reduced, alkylated and trypsinized, for HPLC-MS/MS [18]. Scaffold (version Scaffold_4.0.4, Proteome Software Inc., Portland, OR) was used to validate MS/MS based peptide and protein identifications. Peptide identifications were at greater than $99.0 \%$ probability to achieve an FDR less than $1.0 \%$ [19]. Protein identifications were accepted if greater than $93.0 \%$ probability to achieve an FDR less than $1.0 \%$ (Protein Prophet) [20].

\section{Protoarrays}

Human Protoarrays (Invitrogen) were blocked with 2\% BSA/PBS, rinsed with 0.05\% Tween-20/PBS then incubated with $25 \mu \mathrm{g} / \mathrm{ml} \mathrm{mAb}$ in $2 \%$ BSA/PBS. Bound antibody was detected with Cy5-conjugated goat anti-human $\operatorname{IgG}(0.5 \mu \mathrm{g} / \mathrm{ml}$, Jackson) and counter-stained with rabbit anti-GST (Millipore) followed by Cy3-conjugated goat anti-rabbit $(0.5 \mu \mathrm{g} / \mathrm{ml}$, Jackson). Antigen signals (MFIs) were quantified using a GenePix 4000B microarray scanner and GenePix Pro 6.0 software (Molecular Devices).

Using raw values for the MFI of each probe spot for Cy5 (F635) and Cy3 (F532), and of the probe background for Cy5 (B635) and Cy3 (B532), initial probe intensities were computed as (F635/B635)/(F532/B532). Raw probe intensities were normalized to internal controls and linearly scaled to median control probe intensity of 1.0. Each array was scanned at two PMT voltages, 500 and 600. At PMT 500, we assessed the ratio and the difference between antibody and control intensities: R500 = A500/C500 and D500=A500-C500 (A=antibody, 
$\mathrm{C}=$ control), respectively; the same was done for PMT 600. The overall probe reactivity was then computed as the harmonic mean: 4/(1/R500+1/R600+1/D500+1/D600).

\section{Vimentin microarrays}

Bovine vimentin (SIGMA $100 \mu \mathrm{g} / \mathrm{ml}$ in $0.4 \mathrm{M} \mathrm{HCl}$ ) was diluted 1:1 with printing buffer (ArrayIt) and spotted (20 features/block, 24 blocks/slide) onto SuperEpoxy glass slides using a SpotBot 3 microarrayer (ArrayIt). Slides were washed with $0.05 \%$ Tween-20/PBS and then incubated in BlockIt blocking buffer (ArrayIt). Serum (1:50) or mAbs $(100 \mu \mathrm{g} / \mathrm{ml})$ in reaction buffer (ArrayIt) were applied followed by Cy3-conjugated goat anti-human IgG $(0.5 \mu \mathrm{g} / \mathrm{ml})$. Signals were quantified using a GenePix 4000B microarray scanner and GenePix Pro 6.0 software (Molecular Devices). The MFIs for the 20 vimentin features in each probed block were median averaged.

\section{TII mAb staining of kidney tissue}

Fresh frozen biopsy sections $(3 \mu \mathrm{m})$ were adhered to glass slides fixed in acetone (-20ú C), washed in PBS and permeabilized in 0.5\% NP-40/PBS. Slides were blocked in 10\% DS/PBS and probed with TII mAbs directly conjugated to FITC (Pierce). Bound mAb was detected with rabbit anti-FITC antibodies ( $1 \mu \mathrm{g} / \mathrm{ml}$, Life Technologies), followed by Alexa Fluor 488-conjugated goat anti-rabbit antibodies $(0.5 \mu \mathrm{g} / \mathrm{ml}$, Life Technologies). Cell nuclei were counter-stained with Hoechst 33342.

\section{Results}

\section{Characterization of in situ expressed immunoglobulins}

In situ antigen-driven B cell selection is evident in LuN including intrarenal GCs, T:B cell aggregates and plasmablast foci [13]. All of these histological features contain Ki-67+ $\mathrm{B}$ cells and/or plasmablasts. To characterize the antigen(s) driving in situ B cell selection in each of these tertiary lymphoid-like structures, we identified a cohort of eight lupus patients (Supplementary Table 1) with TII on diagnostic biopsy. Seven of these biopsies had T:B aggregates and plasma cells while one had a GC.

To identify in situ selected B cell populations, five of the biopsies with T:B aggregates and plasma cells (patients 1-5) were sectioned and stained for Ki-67. The biopsy containing a GC (patient 6), and one biopsy with T:B aggregates and plasma cells (patient 7), were stained for CD38. Positive cells were laser captured. From each capture, expressed IgG heavy, lambda or kappa variable $\left(\mathrm{V}_{\gamma}, \mathrm{V}_{\lambda}\right.$ or $\left.\mathrm{V}_{\kappa}\right)$ regions were amplified and subcloned into TA vectors [13]. Multiple colonies from each transformation were sequenced. A particular variable chain sequence was considered valid if the same sequence was obtained from at least two separate colonies of a given transformation. $A V_{H}$ and $V_{L}$ pair was considered valid if the same immunoglobulin heavy chain rearrangement was obtained from at least two different captured areas from one biopsy. Using this approach, 18 paired $\mathrm{V}_{\mathrm{H}}$ and $\mathrm{V}_{\mathrm{L}}$ regions were identified (Supplementary Table 2).

For most patient samples, the above approach produced single $\mathrm{V}_{\mathrm{H}}$ and $\mathrm{V}_{\mathrm{L}}$ pairings. However, in one instance (patient 4) two heavy and two light chains persistently co- 
occurred. In this case, all four possible pairings were considered and expressed as respective mAbs (Ki4-1 through Ki4-4). Additionally, three of the cloned heavy chains persistently cooccurred with two light chains leading us to generate two different TII mAbs for each respective heavy chain (patient 2, Ki2-1 and Ki2-2; patient 3, Ki3-1 and Ki3-2; patient 6, $\mathrm{GC} 1$ and $\mathrm{GC} 1 \mathrm{a})$.

Finally, one biopsy was digested and single CD $38^{+}$cells were sorted by FACS [15]. The variable regions were again subcloned into TA vectors and sequenced. Seven distinct $\mathrm{V}_{\mathrm{H}}$ and $\mathrm{V}_{\mathrm{L}}$ pairings were isolated.

A summary of the nucleotide and predicted amino acid sequences for individual $\mathrm{V}_{\mathrm{H}}$ and $\mathrm{V}_{\mathrm{L}}$ regions is provided in Supplementary Table 2 . The $\mathrm{V}_{\mathrm{H}}$ segments were different for each antibody chain, with the exception that both Ki5-1 and PB1 used $\mathrm{V}_{\mathrm{H}} 3-15$ but with different $\mathrm{D}$ and $\mathrm{J}_{\mathrm{H}}$ segments. Overall, the frequency of $\mathrm{V}_{\mathrm{H}}$ segments was similar to $\mathrm{V}_{\mathrm{H}}$ usage in the peripheral $\mathrm{B}$ cells of healthy populations [21], including a bias for $\mathrm{V}_{\mathrm{H}} 3(12 / 20)$ and $\mathrm{V}_{\mathrm{H}^{4}}$ (7/20). The length of the $\mathrm{V}_{\mathrm{H}}$ complementarity determining region 3 (H-CDR3) and $\mathrm{V}_{\mathrm{L}}$ CDR3 (L-CDR3) varied from 7-23 and 8-12 amino acids respectively. All immunoglobulin heavy and light chains were somatically mutated (Supplementary Table 2) and a distribution of replacement and silent mutations consistent with antigen-driven selection was evident in 13 of $20 \mathrm{~V}_{\mathrm{H}}$ chains (Supplementary Table 3) [16].

\section{Antibodies from in situ selected B cells often recognize cytoplasmic antigens}

Paired $\mathrm{V}_{\mathrm{H}}$ and $\mathrm{V}_{\mathrm{L}}$ regions were subcloned and expressed as functional human $\operatorname{IgG}_{1} \mathrm{mAbs}$. In total, $25 \mathrm{TII}$ mAbs were derived from eight patients; 14 from $\mathrm{Ki}-67^{+}$positive cells, three from $\mathrm{CD} 38^{+}$cells within a $\mathrm{GC}$, one from $\mathrm{CD} 38^{+}$cells within a $\mathrm{T}: \mathrm{B}$ aggregate, and seven from a biopsy sorted for $\mathrm{CD}_{3} 8^{+}$cells (Supplementary Tables 1 and 2).

All 25 TII mAbs were assayed for nuclear reactivity by IIF on HEp-2 cells (Figure 1A-C). Only four reacted preferentially with nuclear antigens; three yielded speckled patterns and one yielded a nucleolar pattern. In contrast, the control human IgG1 mAb, 3H9, which preferentially binds dsDNA, yielded a homogeneous nuclear staining pattern, and reacted to a lesser extent with the cytoplasm.

Eleven antibodies, from seven of the eight patients, bound cytoplasmic antigens. Of these, seven had preferential cytoskeletal reactivity, two yielded a diffuse homogeneous cytoplasmic pattern and two had both cytoplasmic and nuclear binding. One mAb (PB7) colocalized with the golgi apparatus protein giantin (Supplementary Figure S1) [22]. Nine of $25 \mathrm{mAbs}$ demonstrated no detectable reactivity. These data suggest that TII in situ adaptive immune responses are more often targeted to cytoplasmic than nuclear self-antigens.

\section{TIl antibodies do not frequently bind usual nuclear antigens}

To confirm the absence of dsDNA reactivity, Crithidia luciliae were stained with each $\mathrm{mAb}$ (Supplementary Figure S2). While 3H9 had strong reactivity with the kineticore, there was no detectable reactivity with the TII mAbs. ELISAs were subsequently performed for ANA, SSA and Sm/RNP reactivity (Supplementary Figure S3). Interestingly, none of the four nuclear reactive mAbs (PB2, Ki1-2, Ki4-2 and Ki5-3) showed strong reactivity with any of 
these substrates. Ki4-2 did however have low reactivity with Sm/RNP. These data suggest that the nuclear antigen specificities normally associated with SLE are not commonly selected in situ in lupus TII. Furthermore, none of the TII antibodies had appreciable IgG rheumatoid factor activity (Supplementary Figure 3).

\section{Vimentin is an autoantigen commonly targeted in situ}

We next sought to identify the antigenic targets of antibodies with cytoplasmic and cytoskeletal reactivities (both collectively grouped as anti-cyto antibodies). We first interrogated Protoarrays (Life Technologies), containing 9400 human proteins, with a selected group of TII mAbs (Figure 2). The measures of respective antigen reactivities were computed as harmonic mean ratios (see Materials and Methods). We validated the Protoarrays with a transglutaminase 2 (TG2) reactive human $\operatorname{IgG}_{1} \mathrm{mAb}$ (4B06), cloned from a patient with Celiac disease [23]. The two TG2 antigens (IVGN:PM_2144 and BC003551) were the most reactive features, giving values of 32.8 and 11.7 respectively.

The TII mAbs used for Protoarray probing provided at least one example of each staining pattern; anti-cyto (Ki5-2, Ki3-2, Ki3-1, Ki5-1), speckled nuclear (Ki1-2), GA (PB7), nucleolar (Ki5-3) and no appreciable HEp-2 reactivity (GC1, GC1a, PB6, Ki2-1, PB1). A subset of antigens was hierarchically clustered using a Pearson correlation distance measure and displayed in a heat-map (Figure 2); the antigens selected were those with reactivity greater than two-fold for the $99 \%$ value of the control probes. The most commonly reactive targets included fms-related tyrosine kinase 3 ligand (FLT-3LG), IgG1 and IgM heavy chains, and epidermal growth factor (EGF)-like discoidin I-like domains 3 (EDIL3).

Essentially all of the antibodies bound FLT-3LG and therefore this immunospecificity was likely spurious.

Most TII mAbs, comparably to the TG2 specific mAb, displayed restricted poly-reactivity with eight or fewer proteins. In contrast, Ki3-1 displayed greater polyreactivity. Antigens targeted by at least two anti-cyto TII mAbs included EDIL3 and vimentin. In contrast, the nuclei reactive mAb, Ki1-2, only bound weakly to a few Protoarray antigens. A similar pattern of pauci-reactivity was observed with those TII mAbs that did not react with HEp-2 cells.

We next used mass spectrometry to characterize the antigens targeted by the TII anti-cyto mAbs (Figure 3A-B). HEp-2 cell lysates were immunoprecipitated with a mixture of anticyto mAbs (Ki3-1, Ki3-2, Ki5-1, Ki5-2) or a mixture of four different anti-TG2 IgG1 mAbs (4B06, 4A06, 4D03, 4E05) [23]. Lysates were also immunoprecipitated with the antinucleolar mAb Ki5-3 (Supplementary Table 4). Samples were resolved by reducing SDSPAGE, and stained with Coomassie blue (Figure 3A). A few unique bands were detected in the anti-cyto $\mathrm{mAb}$ lane including one with a relative molecular weight of approximately 45 $\mathrm{kDa}(\mathrm{T} 1)$. The $\mathrm{T} 1$ band, and a region corresponding to the same molecular weight from the Ki5-3 immunoprecipitation (T2), were excised for mass spectrometry. From the T1 band, proteins inferred from peptide spectra were cytokeratin-1, -2, -7 and -9, actin, fructose biphosphate aldolase A, and vimentin (Supplementary Table 5). Among the above proteins identified from T1, the cytokeratins were also observed in T2. Cross-referencing of the T1 proteins identified by mass spectrometry to those on the protoarray heat map (Figure 2) 
revealed that only vimentin was common. The two vimentin peptides identified by mass spectrometry (Figure 3B and Supplementary Tables 4 and 5) represented 29 of the 466 amino acids $(6 \%)$ in the full-length translated protein.

Additional immunoprecipitations were performed with a different mixture of cytoskeleton staining TII mAbs (GC2, Ki4-5, PB4 and PB5) using lysates of HEp-2 or primary renal proximal epithelial cells (RPECs). As full-length vimentin has a relative molecular weight of $54 \mathrm{kDa}$, gel tranches covering 50-55 kDa were excised (Supplementary Table 5, tranches T3 and T4 respectively) for mass spectrometry. Vimentin peptides were once again identified in both samples. The lower molecular weight forms of vimentin detected in T1 likely represent proteolytic fragments [24, 25]. In total, 27 different vimentin peptides covering 259 amino acids (56\% of the full length protein) (Figure 3B) were identified from T1, T3, and T4 (Supplementary Table 5). To confirm that vimentin was targeted by TII anti-cyto mAbs, HEp-2 cells were co-stained with the TII mAb Ki3-2 and the murine anti-vimentin mAb V9 (DAKO, Figure 3C), revealing extensive co-localization.

To determine whether vimentin was a frequent target of antibodies produced in situ during TII, all 10 remaining anti-cyto TII mAbs were assayed by confocal microscopy. All 10 had some overlap with V9 staining, with PB4, Ki3-1, GC2, PB3, PB5, and Ki1-1 showing extensive and specific overlap (Figure 4A and Supplementary Figures 4 and 5). Polyreactivity was observed with the TII mAbs Ki2-2, Ki5-1 and Ki5-2, (Supplementary Figures 4 and 5), while Ki4-5 (Figure 4A) demonstrated preferential staining of cytoskeletal filaments, some of which did not react with V9. As these co-localizations could represent binding to antigens closely neighboring vimentin, direct vimentin immunoreactivity was confirmed using purified bovine vimentin coated Superepoxy glass slides (Figure 4B). Ten of 11 anti-cyto TII mAbs had vimentin immunoreactivity that was greater than that observed for any of the other non-cyto TII mAbs $\left(p=3.9 \times 10^{-4}\right)$.

\section{Vimentin is upregulated in inflamed tubulointerstitium}

To begin to understand why vimentin was so commonly targeted in situ, we examined the distribution of vimentin expression in normal and LuN renal biopsies by immunohistochemistry (Figure 4C). Consistent with previous reports [24, 26], in normal kidney, vimentin is expressed primarily in glomeruli, arterioles, and interstitial fibroblasts, but not in epithelial cells. In contrast to normal kidney, the distribution and intensity of vimentin expression in TII was radically different, with vimentin being abundant throughout the tubulointerstitium. Much of this increased vimentin expression localized to the mononuclear cells infiltrating the tubulointerstitium.

High expression of vimentin by infiltrating mononuclear cells suggested that in situ humoral immunity is directed against molecules associated with inflammation. Therefore, we directly examined whether TII mAbs also preferentially bound inflamed tubulointersitium (Figure 5). Two TII mAbs, Ki3-1 and PB4, both with vimentin immunoreactivity, were directly labeled with FITC and used to stain sections from normal kidney and LuN kidney with severe TII. Samples were then incubated with rabbit anti-FITC antibodies followed by Alexa Fluor 488-conjugated goat anti-rabbit antibodies (Supplementary Figure 6). As expected, no discernable immunoreactivity was detected in the absence of primary antibody (Figure 5). In 
contrast, staining with $3 \mathrm{H} 9$ yielded a predictable nuclear pattern. In normal kidney, Ki3-1 had some immunoreactivity with glomeruli and minimal immunoreactivity with the tubulointerstitium. PB4 demonstrated little immunoreactivity with normal renal tissue. In contrast, both antibodies bound inflamed tubulointerstitium and Ki3-1 also bound inflamed glomeruli. These data are consistent with a model in which in situ immunity targets inflammation.

\section{Serum anti-vimentin antibodies associated with severe TII}

As vimentin is frequently targeted by the in situ humoral immune response, it was predicted that serum anti-vimentin antibody (AVA) titers would reflect TII severity. Therefore, AVA titers in serum samples from patients with $\mathrm{LuN}(\mathrm{n}=48)$, or with no history of renal disease $(n=35)$ were assayed as above (Figure 6A-B). High titer AVAs (>180 AU) were almost exclusively found in patients with renal disease, and renal patients had higher average AVA titers $(p=0.01)$. The one non-renal patient who had a relatively high AVA titer had very active extra-renal disease with severe discoid lupus, arthritis, vasculitis, decreased serum complements and increased anti-dsDNA antibody titers. Within the renal group, high-titer AVAs strongly correlated with severe (grade 3 ) TII $(p<0.0001)$ with the five highest AVA titers detected within this group. Only one of 10 patients with severe TII had low titer AVAs. These data suggest that high-titer AVAs might be a useful biomarker of severe TII.

\section{Discussion}

Many studies have first identified autoantibody specificities in serum and have subsequently correlated these to disease manifestations [2, 27]. Instead, our study started with the affected end organ, providing immediate pathological context. The relevance of the characterized insitu derived anti-vimentin mAbs to TII was supported by the correlation between high serum AVA titers and severe TII. Our results suggest that tissue-focused studies can identify clinically important biomarkers, and potentially pathogenic mechanisms, not immediately apparent from peripheral blood studies.

In Lupus TII, in situ humoral immunity appeared antigenically restricted and primarily focused on cytoplasmic antigens, notably vimentin. Vimentin was expressed by infiltrating inflammatory cells and tested TII mAbs preferentially reacted with the inflamed tubulointerstitium. We postulate that by targeting inflammation the adaptive immune response, and the attendant deposition of antibodies and complement, feed-forward to amplify local inflammation, tissue destruction and renal scarring [28].

Of the other serological specificities associated with SLE, only anti-dsDNA antibody titers provide a similar correlation with disease activity $[29,30]$. This is best defined for GN, where high-titer dsDNA antibodies predict renal flare in some patients [2] and anti-dsDNA antibodies can be detected deposited in inflamed glomeruli [9,31]. While dsDNA antibody titers provide a measure of systemic autoimmunity, we suggest that AVA titers provide complementary measures of organ intrinsic mechanisms of autoimmunity.

Most of the identified antigens targeted by the in situ humoral immune response were cytosolic proteins, the immune response to which usually requires T-cell help [32]. Indeed, 
$\mathrm{T}$ follicular helper $\left(\mathrm{T}_{\mathrm{FH}}\right)$ cells are common in LuN biopsies manifesting severe TII, where they are in apparent cognate pairs with B cells [33]. Therefore, the inflamed tubulointerstitium appears sufficient to propagate cytosolic antigen targeted humoral immunity. In contrast, inflamed glomeruli lack infiltrating $\mathrm{B}$ and $\mathrm{T}_{\mathrm{FH}}$ cells. B cell expressed toll-like receptors might therefore be less important in situ than they are in systemic immune responses measured in the periphery (and propagated in secondary lymphoid organs), where these receptors play a role in responses to dsDNA and RNP containing complexes [34, 35].

Some characteristics of vimentin might enhance its antigenicity in situ. Vimentin is a highly basic molecule that is secreted by activated macrophages [25]. Therefore, in inflammation, vimentin is abundant and available. Furthermore, vimentin can bind and activate dectin-1 [36], a c-type lectin receptor expressed on cells including macrophages, dendritic cells, and B cells. Vimentin might therefore locally prime antigen-presenting cells. Finally, vimentin can be post-translationally modified which, in RA, provides neo-epitopes and increases antigenicity [37]. It remains to be determined if post-translational modifications enhance in situ immunity to vimentin in LuN TII, or whether particular HLA molecules preferentially present vimentin-derived peptides. Mapping dominant vimentin epitopes should provide insights into these questions.

Most AVAs demonstrated polyreactivity. This could reflect a less efficient selection on multiple abundant antigens in the inflamed kidney, in comparison to a predictably stringent selection in GCs, in which antigen is limited and presented in competitive contexts that favor higher affinity and restricted polyreactivity [38]. Alternatively, the heightened polyreactivity/poly-specificity may be due to more than one antigen driving selection, reflecting poly-selection.

Interestingly, anti-cytoplasmic antibodies are a common feature of the normal newly emigrated and mature naïve B cell repertoires [39]. Furthermore, in SLE anti-cytoplasmic antibodies are even more frequent in these pools with at least a third of mature naive B cells having some cytoplasmic reactivity [40]. The high prevalence of B cells expressing anticytoplasmic antibodies may help explain the low-titer AVAs observed in most SLE patients. Presumably, the TII AVAs that we characterized arose from one or more of the above B cell populations expressing anti-cytoplasmic antibodies. Alternatively, it is possible that the AVAs were selected from non-autoreactive or anti-nuclear antigen specific B cell precursor populations.

High-titer AVAs (>225 AU) were invariably associated with high disease activity, usually severe TII. Among the six highest AVA titers, five occurred in severe TII with all four of the very highest titers associated with this manifestation. The only non-nephritic patient with high-titer AVAs (236 AU) had severe extra-renal disease. Intermediate AVA titers (150-220 AU) were preferentially found in patients with nephritis. Therefore, different titers of AVAs might reflect different levels of disease activity and disease manifestations.

Anti-cytoplasmic antibodies and AVAs have been observed in a variety of autoimmune diseases [41-44] and in transplant rejection. Interestingly, in cardiac allograft recipients, IgM AVAs are predictive of allograft vasculopathy [45] and rejection [46]. Furthermore, 
AVAs have been observed in renal transplant rejection [47, 48]. In mice, immunization with vimentin accelerates cardiac allograft rejection [49] providing evidence that anti-vimentin immune responses can be pathogenic. These data, with ours, suggest that AVAs represent a common adaptive immune response in chronic organ inflammation which perpetuates disease.

Because of the difficulties inherent in isolating in situ selected B cells, our study was restricted to a small number of biopsies and mAbs. Sampling single cells by LCM is imprecise and it is likely that a few $\mathrm{V}_{\mathrm{H}}$ and $\mathrm{V}_{\mathrm{L}}$ pairings were spurious. Indeed, in some cases a single $\mathrm{V}_{\mathrm{H}}$ could not be paired with a single $\mathrm{V}_{\mathrm{L}}$. Furthermore, closed needle biopsies provide a limited sampling of the entire lesion. Despite these limitations, our study revealed consistent themes; antibodies from seven of eight patients were immunoreactive with cytoplasmic antigens and antibodies from six of eight patients had vimentin immunoreactivity. Furthermore, these prevalences correlated with the frequency of high serum AVA titers in patients with severe TII. Therefore, our sample size and approach appeared sufficient to capture common features of the TII in situ adaptive immune response.

This study demonstrates that high AVA titers identify lupus patients with severe TII, a population at substantial risk for progression to renal failure $[6,7,12]$. Whether AVAs are directly pathogenic, or if they can be used as a biomarker, requires additional study. Also, it is not known if high AVA titers identify responders to targeted therapies. Regardless, knowing where and when AVAs arise in lupus will provide a strong context for interpreting future clinical and mechanistic studies.

\section{Supplementary Material}

Refer to Web version on PubMed Central for supplementary material.

\section{Acknowledgments}

We would like to thank Christine Labno and Margaret Veselits for technical assistance with confocal microscopy. Justin Jarrel provided technical assistance with antigen microarrays while Keith Hamel and Sophiya Karki critically read the manuscript.

Funding:

Funding was provided by the NIH (AR55646) and NIH Autoimmunity Centers of Excellence (AI082724). DJH is funded by a Canadian Institutes for Health Research (CIHR) Fellowship.

\section{References}

1. Liu Z, Davidson A. Taming lupus-a new understanding of pathogenesis is leading to clinical advances. Nat Med. 2012; 18:871-882. [PubMed: 22674006]

2. Lahita, RG.; Tsokos, G.; Buyon, JP.; Koike, T. Systemic Lupus Erythematosus. 4. San Diego: Elsevier Academic Press; 2010.

3. Mok C, Tang SS. Incidence and predictors of renal disease in Chinese patients with systemic lupus erythematosus. Am J Med. 2004; 117(10):791-795. [PubMed: 15541328]

4. Cervera R, Khamashta MA, Font J, Sebastiani GD, Gil A, Lavilla P, Mejia JC, Aydingtug AO, Chwalinska-Sadowska H, de Ramon E, Fernandez-Nebro A, Galeazzi M, Valen M, Mathieu A, Houssiau F, Caro N, Alba P, Ramos-Casals M, Ingelmo M, Hughes GR. European Working Party on Systemic Lupus Erythematosus: Morbidity and mortality in systemic lupus erythematosus during 
a 10-year period: a comparison of early and late manifestations in a cohort of 1,000 patients. Medicine (Baltimore). 2003; 82(5):299-308. [PubMed: 14530779]

5. Ginzler E, Dooley MA, Aranow C, Kim MY, Buyon J, Merrill JT, Petri, Gilkeson S, Wallace DJ, Weisman MH, Appel GB. Mycophenolate mofetil or intravenous cyclophosphamide for lupus nephritis. N Engl J Med. 2005; 353(21):2219-2228. [PubMed: 16306519]

6. Esdaile JM, Levinton C, Federgreen W, Hayslett JP, Kashgarian M. The clinical and renal biopsy predictors of long-term outcome in lupus nephritis: a study of 87 patients and review of the literature. Q J Med. 1989; 72(269):779-833. [PubMed: 2694209]

7. Hsieh C, Chang A, Brandt D, Guttikonda R, Utset TO, Clark MR. Predicting outcomes of lupus nephritis with tubulointerstitial inflammation and scarring. Arthritis Care Res (Hoboken). 2011; 63(6):865-874. [PubMed: 21309006]

8. Ebling F, Hahn BH. Restricted subpopulations of DNA antibodies in kidneys of mice with systemic lupus. Comparison of antibodies in serum and renal eluates. Arth Rheum. 1980; 23 (4):392-403. [PubMed: 7370058]

9. Winfield JB, Faiferman I, Koffler D. Avidity of anti-DNA antibodies in serum and IgG glomerular eluates from patients with systemic lupus erythematosus. Association of high avidity antinative DNA antibody with glomerulonephritis. J Clin Invest. 1977; 59:90-96. [PubMed: 299748]

10. Ehrenstein MR, Katz DR, Griffiths MH, Papadaki L, Winkler TH, Kalden JR, Isenberg DA. Human IgG anti-DNA antibodies deposit in kidneys and induce proteinuria in SCID mice. Kid Int. 1995; 48(3):705-711.

11. Madaio MP, Carlson J, Cataldo J, Ucci A, Migliorini P, Pankewycz O. Murine monoclonal antiDNA antibodies bind directly to glomerular antigens and form immune deposits. J Immunol. 1987; 138:2883-2889. [PubMed: 3553329]

12. Yu F, Wu LH, Tan Y, Li L-H, Wang C-L, Wang C-L, Wang W-K, Qu Z, Chen MA, Gao JJ, et al. Tubulointerstitial lesions of patients with lupus nephritis classified by the 2003 international society of nephrology and renal pathology society system. Kid Int. 2010; 77:820-829.

13. Chang A, Henderson SG, Liu N, Guttikonda R, Hsieh C, Utset TO, Meehan SM, Quigg RJ, Meffre E, Clark MR. In situ B cell-mediated immune responses and tubulointerstitial inflammation in human lupus nephritis. J Immunol. 2011; 186(3):1849-1860. [PubMed: 21187439]

14. Davidson A, Aranow C. Lupus nephritis: lessons from murine models. Nat Rev Immunol. 2010; 6:13-20.

15. Smith K, Garman L, Wrammert J, Zheng NY, Capra JD, Ahmed R, Wilson PC. Rapid generation of fully human monoclonal antibodies specific to a vaccinating antigen. Nat Protoc. 2009; 4:372384. [PubMed: 19247287]

16. Lossos IS, Tibshirani R, Narasimhan B, Levy R. The inference of antigen selection on Ig genes. J Immunol. 2000; 165(9):5122-5126. [PubMed: 11046043]

17. O’Neill SK, Veselits ML, Zhang M, Labno C, Cao Y, Finnegan A, Uccelini M, Alegre ML, Cambier JC, Clark MR. Endocytic sequestration of the B cell antigen receptor and toll-like receptor 9 in anergic B cells. Proc Nat Acad Sci, USA. 2009; 106:6262-6267. [PubMed: 19332776]

18. Truman AW, Kristjansdottir K, Wolfgeher D, Hasin N, Polier S, Zhang H, Perrett S, Prodromou C, Jones GW, Kron SJ. CDK-dependent Hsp70 Phosphorylation controls G1 cyclin abundance and cell-cycle progression. Cell. 2012; 151:1308-1318. [PubMed: 23217712]

19. Keller A, Nesvizhskii AI, Kolker E, Aebersold R. Empirical statistical model to estimate the accuracy of peptide identifications made by MS/MS and database search. Anal Chem. 2002; 74:5382-5392.

20. Nesvizhskii AI, Keller A, Kolker E, Aebersold R. A statistical model for identifying proteins by tandem mass spectrometry. Anal Chem. 2003; 75:4646-4658. [PubMed: 14632076]

21. Brezinschek HP, Brezinschek RI, Lipsky PE. Analysis of the heavy chain repertoire of human peripheral B cells using single-cell polymerase chain reaction. J Immunol. 1995; 155(1):190-202. [PubMed: 7602095]

22. Nozawa K, Fritzler MJ, von Muhlen CA, Chan EK. Giantin is the major Golgi autoantigen in human anti-Golgi complex sera. Arthritis Res Ther. 2004; 6(2):R95-102. [PubMed: 15059272] 
23. Di Niro R, Mesin L, Zheng NY, Stamnaes J, Morrissey M, Lee JH, Huang M, Iversen R, du Pre MF, Qiao SW, et al. High abundance of plasma cells secreting transglutaminase 2-specific IgA autoantibodies with limited somatic hypermutation in celia disease interstinal lesions. Nat Med. 2012; 18:441-451. [PubMed: 22366952]

24. Buchmaier BS, Bibi A, Muller GA, Dihazi GH, Eltoweissy M, Kruegel J, Dihazi H. Renal cells express different forms of vimentin: the independent expression alteration of these forms is important in cell resistance to osmotic stress and apoptosis. PLoS ONE. 2013; 8(7):e68301. [PubMed: 23874579]

25. Mor-Vaknin N, Punturieri A, Sitwala K, Markovitz DM. Vimentin is secreted by activated macrophages. Nat Cell Biol. 2003; 5:59-63. [PubMed: 12483219]

26. de Matos AC, Camara NO, Tonato EJ, de Durao MS Junior, Franco MF, Moura LA, Pacheco-Silva A. Vimentin expression and myofibroblast infiltration are early markers of renal dysfunction in kidney transplantation: an early stage of chronic allograft dysfunction? Transplant Proc. 2010; 42(9):3482-3488. [PubMed: 21094801]

27. Tan EM, Kunkel HG. Characteristics of a soluble nuclear antigen precipitation with sera of patients with systemic lupus erythematosus. J Immunol. 1966; 96:464-471. [PubMed: 5932578]

28. Liu L, Kou P, Zeng Q, Pei G, Li Y, Liang H, Xu G, Chen S. CD4+ T lymphocytes, especially Th2 cells, contribute to the progress of renal fibrosis. Am J Nephrol. 2012; 36:386-396. [PubMed: 23052013]

29. Ter Borg EJ, Horst G, Hummel EJ, Limburg PL, Kallenberg CGM. Measurement of increases in anti-double-stranded DNA antibody levels as a predictor of disease exacerbation in systemic lupus erythematosus. Arth Rheum. 1990; 33:634-643. [PubMed: 2346519]

30. Bootsma H, Spronk PE, Ter Borg EJ, Hummel EJ, de Boer G, Limubrg PC, Kallenberg CG. The predictive value of fluctuations in IgM and IgG class anti-dsDNA antibodies for relapses in systemic lupus erythematosus. A prospective long-term observation. Ann Rheum Dis. 1997; 56:661-666. [PubMed: 9462168]

31. Kalaaji M, Fenton KA, Mortensen ES, Olsen R, Sturfelt G, Alm P, Rekvig OP. Glomerular apoptotic nucleosomes are central target structures for nephritogenic antibodies in human SLE nephritis. Kid Int. 2007; 71:664-672.

32. Crotty S. Follicular helper CD4 T cells (TFH). Ann Rev Immunol. 2011; 29:621-663. [PubMed: 21314428]

33. Liarski V, Kaverina N, Chang A, Brand D, Carlesso G, Utset TO, Labno C, Peng Y, Jiang Y, Giger ML, et al. Quantitative cell distance mapping in human nephritis reveals organization of in situ adaptive immune responses. Sci Trans Med. 2014 in press.

34. Shlomchik MJ. Sites and stages of autoreactive B cell activation and regulation. Immunity. 2008; 28:18-28. [PubMed: 18199415]

35. Christensen SR, Shupe J, Nickerson K, Kashgarian M, Flavell RA, Shlomchik MJ. Toll-like receptor 7 and TLR9 dictate autoantibody specificity and have opposing inflammatory and regulatory roles in a murine model of lupus. Immunity. 2006; 25(3):417-428. [PubMed: 16973389]

36. Thiagarajan PS, Yakubenko VP, Elsori DH, Yadav SP, Willard B, Tan CD, Rodriguez ER, Febbraio M, Cathcart MK. Vimentin is an endogenous ligand for the pattern recognition receptor Dectin-1. Cardiovasc Res. 2013; 99(3):494-504. [PubMed: 23674515]

37. Bang H, Egerer K, Gauliard A, Luthke K, Rudolph PE, Fredenhagen G, Berg W, Feist E, Burmester GR. Mutation and citrullination modifies vimentin to a novel autoantigen for rheumatoid arthritis. Arthritis Rheum. 2007; 56(8):2503-2511. [PubMed: 17665451]

38. Shlomchik MJ, Weisel F. Germinal center selection and the development of memory B and plasma cells. Immunol Rev. 2012; 247:52-63. [PubMed: 22500831]

39. Wardemann H, Yurasov S, Schaefer A, Young JW, Meffre E, Nussenzweig MC. Predominant autoantibody production by early human B cell precursors. Science. 2003; 301(5638):1374-1377. [PubMed: 12920303]

40. Yurasov S, Wardemann H, Hammersen J, Tsuiji M, Meffre E, Pascual V, Nussenzweig MC. Defective B cell tolerance checkpoints in systemic lupus erythematosus. J Exp Med. 2005; 201:703-711. [PubMed: 15738055] 
41. Ooka S, Nakano H, Matsuda T, Okamoto K, Suematsu N, Korokawa MS, Ohtani-Kaneko R, Masuko K, Ozaki S, Kato T. Proteomic surveillance of autoantigens in patients with Behcet's disease by a proteomic approach. Microbiol Immunol. 2010; 54:354-361. [PubMed: 20536734]

42. Alcover A, Ramierez-Lafita F, Hernandez C, Nieto A, Avlia J. Antibodies to vimentin intermediate filaments in sera from patients with SLE and RA: quantitation by solid phase radioimmunoassay. J Rheumatol. 1985; 12:233-236. [PubMed: 3897530]

43. Clemente MG, Musu MP, Frau F, Brusco G, Sole G, Corazza GR, De Virgiliis S. Immune reaction against the cytoskeleton in coeliac disease. Gut. 2000; 47:520-526. [PubMed: 10986212]

44. Senecal JL, Oliver JM, Rothfeld N. Anticytoskeletal autoantibodies in connective tissue diseases. Arth Rheum. 1985; 28:889-898. [PubMed: 4040759]

45. Jurcevic S, Ainsworth ME, Pomerance A, Smith JD, Robinson DR, Dunn MJ, Tacoub MH, Rose ML. Antivimentin antibodies are an independent predictor of transplant-associated coronary artery disease after cardiac transplantation. Transplantation. 2001; 71:886-892. [PubMed: 11349721]

46. Nath DS, Ilias BG, Tiriveedhi V, Alur C, Phelan D, Eward GA, NM, Mohanakumar T.

Characterization of immune responses to cardiac self-antigens myosin and vimentin in human cardiac allograft recipients with antibody-mediated rejection and cardiac allograft vasculopathy. $\mathrm{J}$ Heart Lung Transplant. 2010; 29:1277-1285. [PubMed: 20615726]

47. Carter V, Howell WM. Vimentin antibody production in transplant patients and immunodulatory effects of vimentin in vitro. Hum Immunol. 2013; 74:1463-1469. [PubMed: 23777931]

48. Carter V, Shenton BK, Jaques B, Turner D, Talbot D, Gupta A, Chapman CE, Matthews CJ, Cavanagh G. Vimentin antibodies: a non-HLA antibody potential risk factor in renal tranplantation. Transplant Proc. 2005; 37:654-657. [PubMed: 15848491]

49. Mahesh B, Leong JS, McCormak A, Sarathchandra P, Holder A, Rose ML. Autoantibodies to vimentin cause accelerated rejection of cardiac allografts. Am J Pathol. 2007; 170:1415-1427. [PubMed: 17392180] 

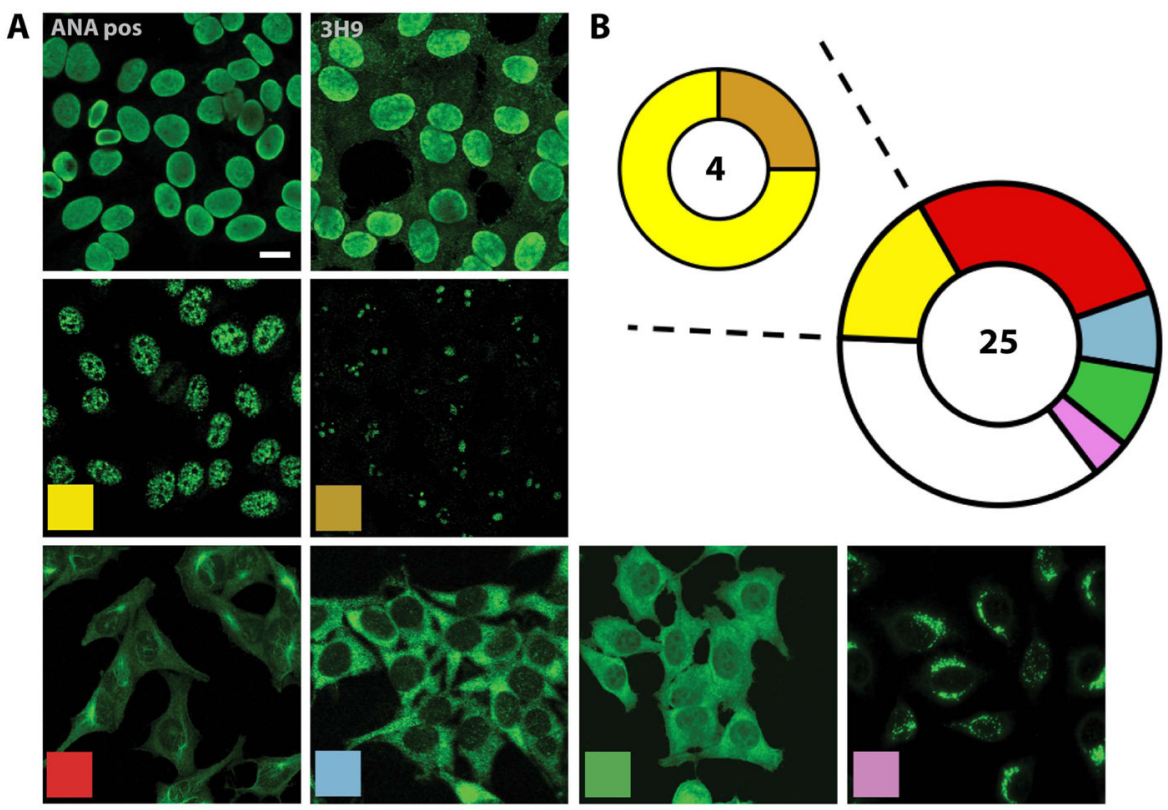

C mAb staining pattern

Ki1-1 cytosk

Ki1-2 nuc spec

Ki2-1 N.R.

Ki2-2 cytopl

Ki3-1 cytosk

Ki3-2 cytosk

Ki4-1 N.R.

Ki4-2 nuc spec

Ki4-3 N.R.

Ki4-4 N.R.

Ki4-5 cytosk

Ki5-1 cytopl \& nuc

Ki5-2 cytopl \& nuc

Ki5-3 nucleolar

GC1 N.R.

GC1a N.R.

GC2 cytosk

TB1 N.R.

PB1 N.R.

PB2 nuc spec

PB3 cytopl

PB4 cytosk

PB5 cytosk

PB6 N.R.

PB7 GA

Figure 1.

HEp-2 staining patterns using TII mAbs. A, Indirect immunofluorescent microscopy of HEp- 2 cells following staining with TII mAbs $(25 \mu \mathrm{g} / \mathrm{ml})$. Provided are representative examples. Positive controls included a known $\mathrm{ANA}^{+}$serum sample (ANA pos) and the human $3 \mathrm{H} 9 \mathrm{IgG} 1 \mathrm{mAb}$, both of which demonstrate a preferential reactivity for DNA and a characteristic homogeneous nuclear staining pattern. White scale bar $=10 \mu \mathrm{m}$. B, Pie charts summarizing relative frequencies of HEp-2 staining patterns observed for TII mAb. The smaller chart is a sub-division of the mAbs that preferentially react with nuclear antigens. Colors correspond to representative images in A. Color key: Yellow: nuclear speckled (nuc spec); brown: nucleolar; red: cytoskeletal (cytosk); blue: cytoplasmic (cytopl); green: cytoplasmic and nuclear (cytopl \& nuc); pink: golgi apparatus (GA); white: no reactivity (N.R.). C, Staining patterns of respective TII mAbs. 


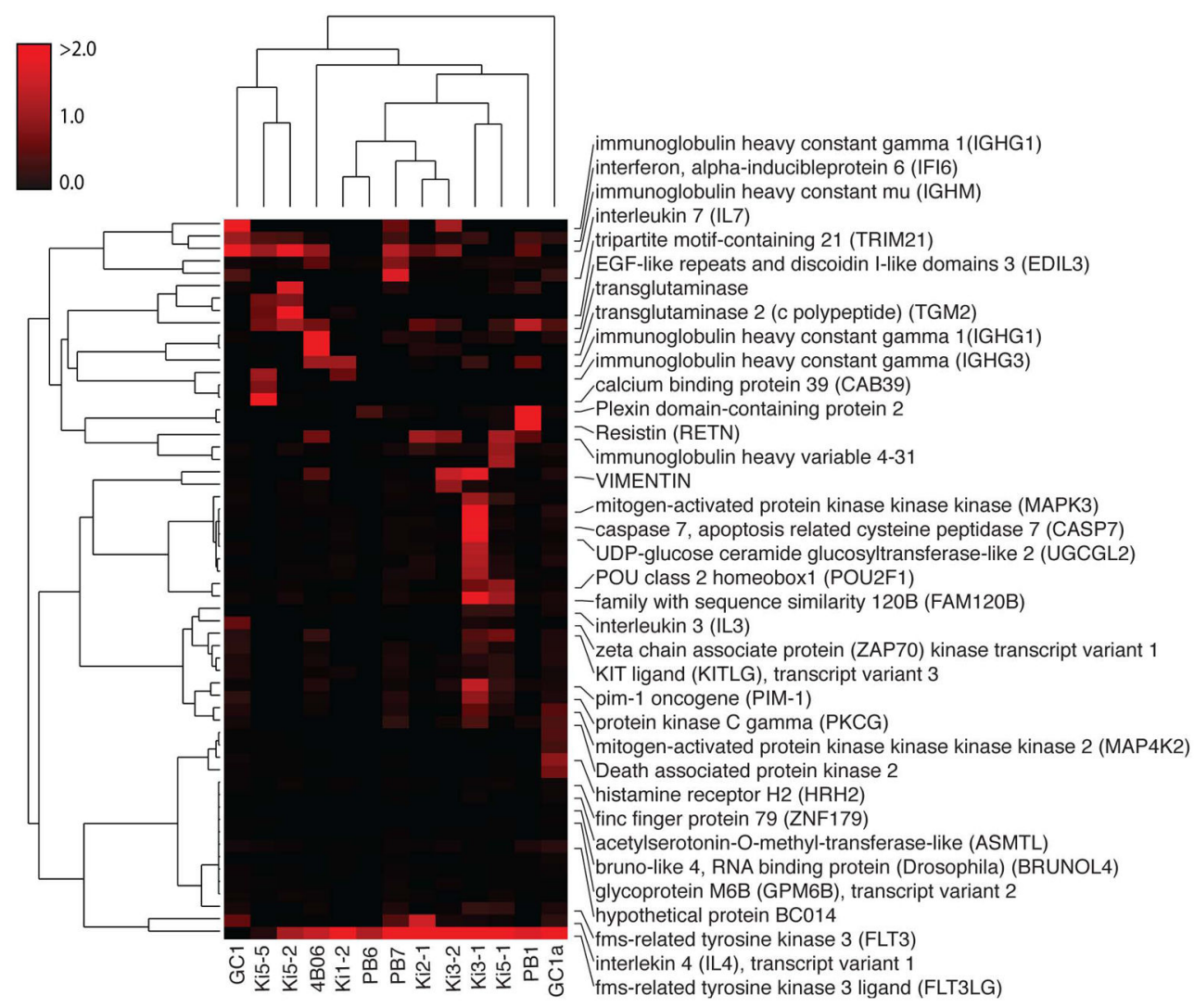

Figure 2.

Immunoreactivities of TII mAbs with Protoarrays. Antibodies with high reactivity in at least one array (defined as at least two-fold higher than $99 \%$ of control probes in the same array) were hierarchically clustered by antigen immunoreactivity. mAb 4B06 has known immunoreactivity with transglutaminase 2 (TG2). The key (top left) indicates the magnitude of immunoreactivity (Materials and Methods). 

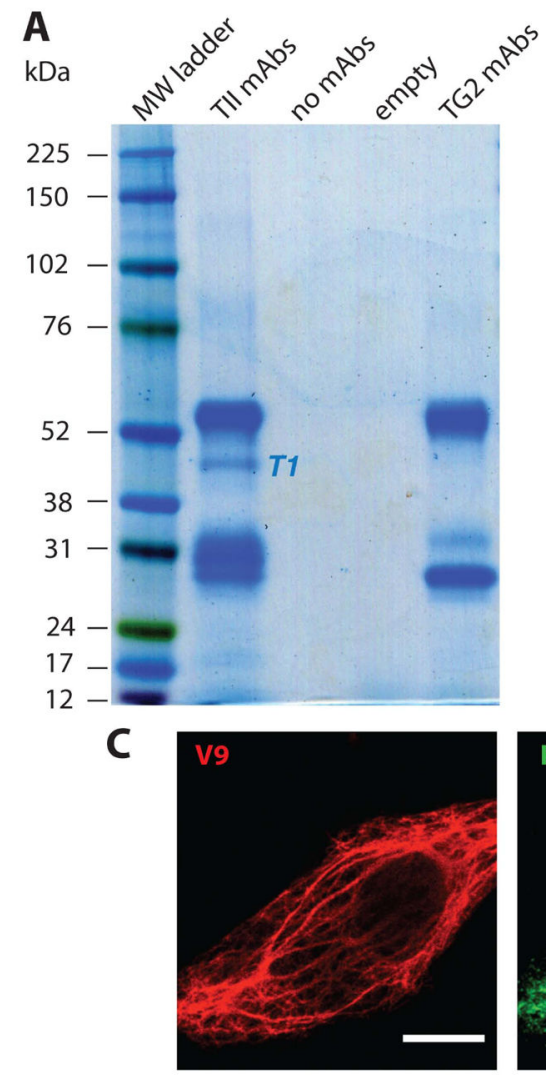

B

vimentin NP_003371.2

53,652.7 Da

MSTRSVSSSSYRRMFGGPGTASRPSSSR SYVTTSTRTYSLGSALRPSTSRSLYASS PGGVYATRSSAVRLRSSVPGVR LLODSV DFSLADAINTEFKNTRTNEKVELQELND RFANYIDKVRFLEQQNKILLAELEQLKG OGKSRLGDLYEEEMRELRROVDOLTNDK ARVEVERDNLAEDIMRLREKLQEEMLQR EEAENTLQSFRQDVDNASLARLDLERKV ESLQEE IAFLKKLHEEE IQELQAQIQEQ HVQIDVDVSKPDLTAALRDVRQQYESVA AKNLQEAEEWYKSKFADLSEAANRNNDA LRQAKQESTEYRRQVQSLTCEVDALKGT NESLERQMREMEENFAVEAANYQDTIGR LQDE IQNMKEEMARHLREYQDLLNVKMA LDIEIATYRKLLEGEESRISLPLPNFSS LNLRETNLDSLPLVDTHSKRTLLIKTVE TRDGQVINETSQHHDDLE

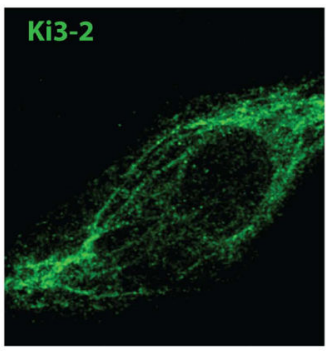

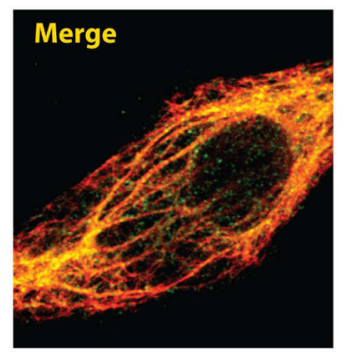

Figure 3.

Identification of vimentin as a putative antigen targeted by TII mAbs. A, Immunoprecipitations of HEp-2 cell lysates with either TII mAbs (Ki3-1, Ki3-2, Ki5-1 and Ki5-2), no mAbs, or TG2 reactive mAbs, were resolved by reducing SDS-PAGE and Coomassie stained. Unique TII mAb band (T1) was excised and analyzed by mass spectrometry. Among proteins identified was vimentin. B, The vimentin (NP_003371.2) amino acid FASTA sequence is italicized and blue in regions corresponding to peptides identified by mass spectrometry of $\mathrm{T} 1$. Tranches cut from analogous immunoprecipitates (Supplementary Tables S4 and S5), generated with other TII mAbs, yielded additional vimentin peptides (bold). C, Confocal microscopy of HEp-2 cells demonstrating co-staining of TII mAb Ki3-2 with anti-vimentin mAb, V9. White scale bar=10 $\mu \mathrm{m}$. 


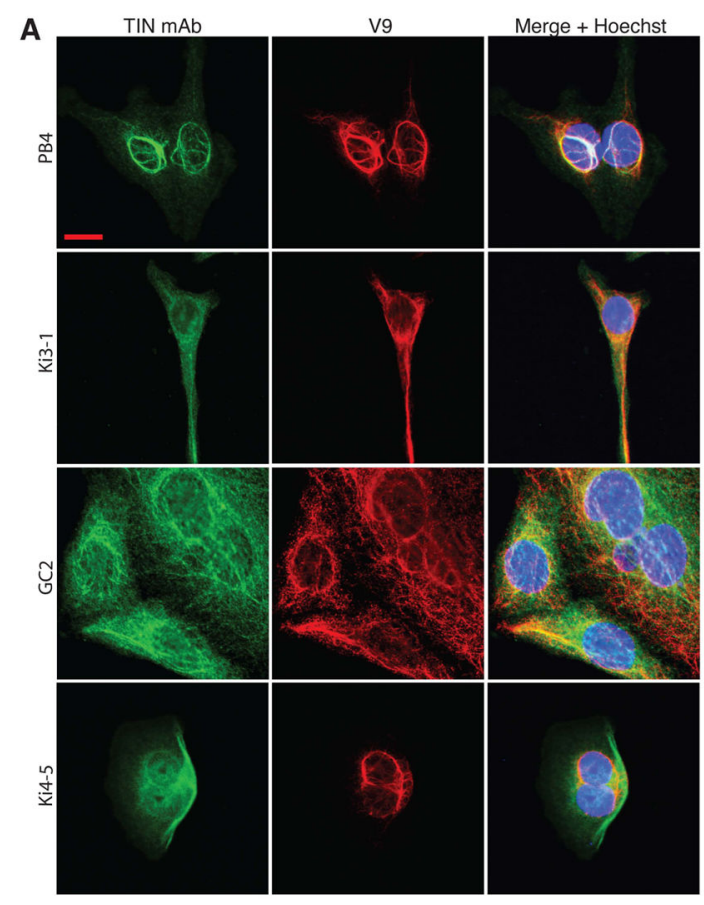

B

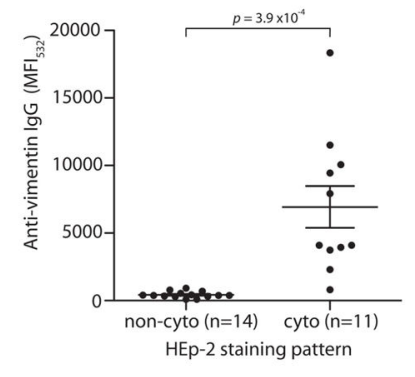

C

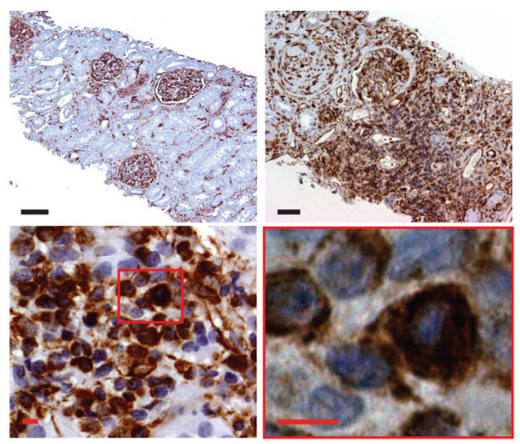

Figure 4.

Vimentin is frequently targeted by TII mAbs. A, HEp-2 cells co-stained with TII mAbs (green), anti-vimentin (V9, red) and Hoechst (blue). Examples of four different patterns of co-localization are shown. Staining with other TII mAbs with cytoplasmic reactivity is provided in Supplementary Figures 4 and 5. B, Vimentin protein arrays probed with TII $\mathrm{mAbs}$, detected with anti-hIgG $\mathrm{H}_{532}$. Data-points are reactivities of individual TII mAbs. Raw MFIs are given. mAbs are grouped by cytoplasmic (cyto) HEp-2 immunoreactivity or not (Mann-Whitney two-tailed test indicated). C, Immunohistochemical staining of normal (top left) and TII (other panels) kidney tissue with V9 antibody (DAKO). Red box: area magnified. Red and black scale bars: $10 \mu \mathrm{m}$ and $100 \mu \mathrm{m}$ respectively. 


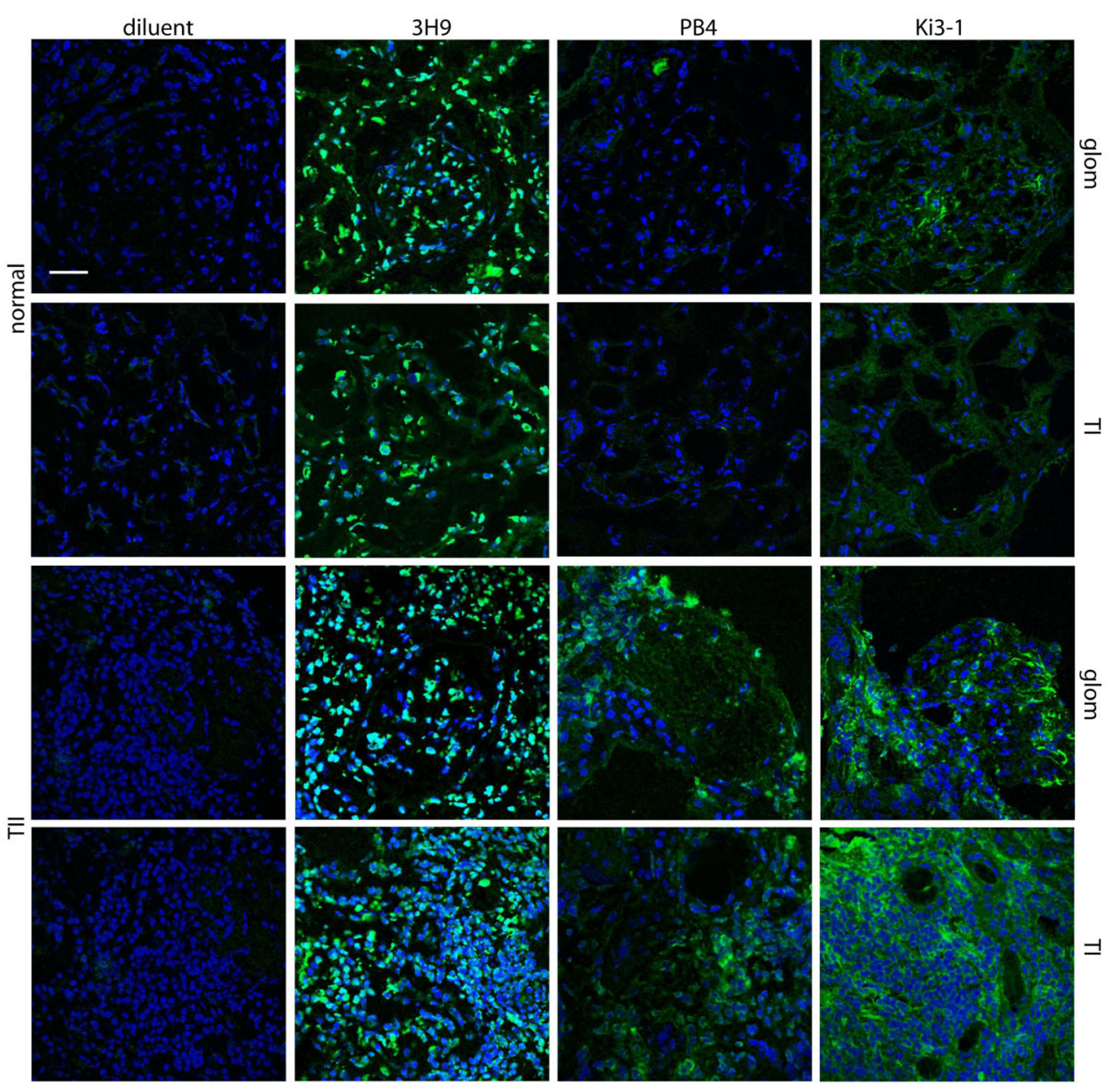

Figure 5.

Enhanced binding of TII mAbs to inflamed renal tubulointerstitium. Indicated TII mAbs were used to stain normal renal and TII samples. The mAb $3 \mathrm{H} 9$ and diluent were used as positive and negative controls respectively. Tissue was counter-stained with Hoechst for nuclei (blue). Representative patterns of tubulointerstitial (TI) and glomerular (glom) binding are given. White scale $b a r=10 \mu \mathrm{m}$. 

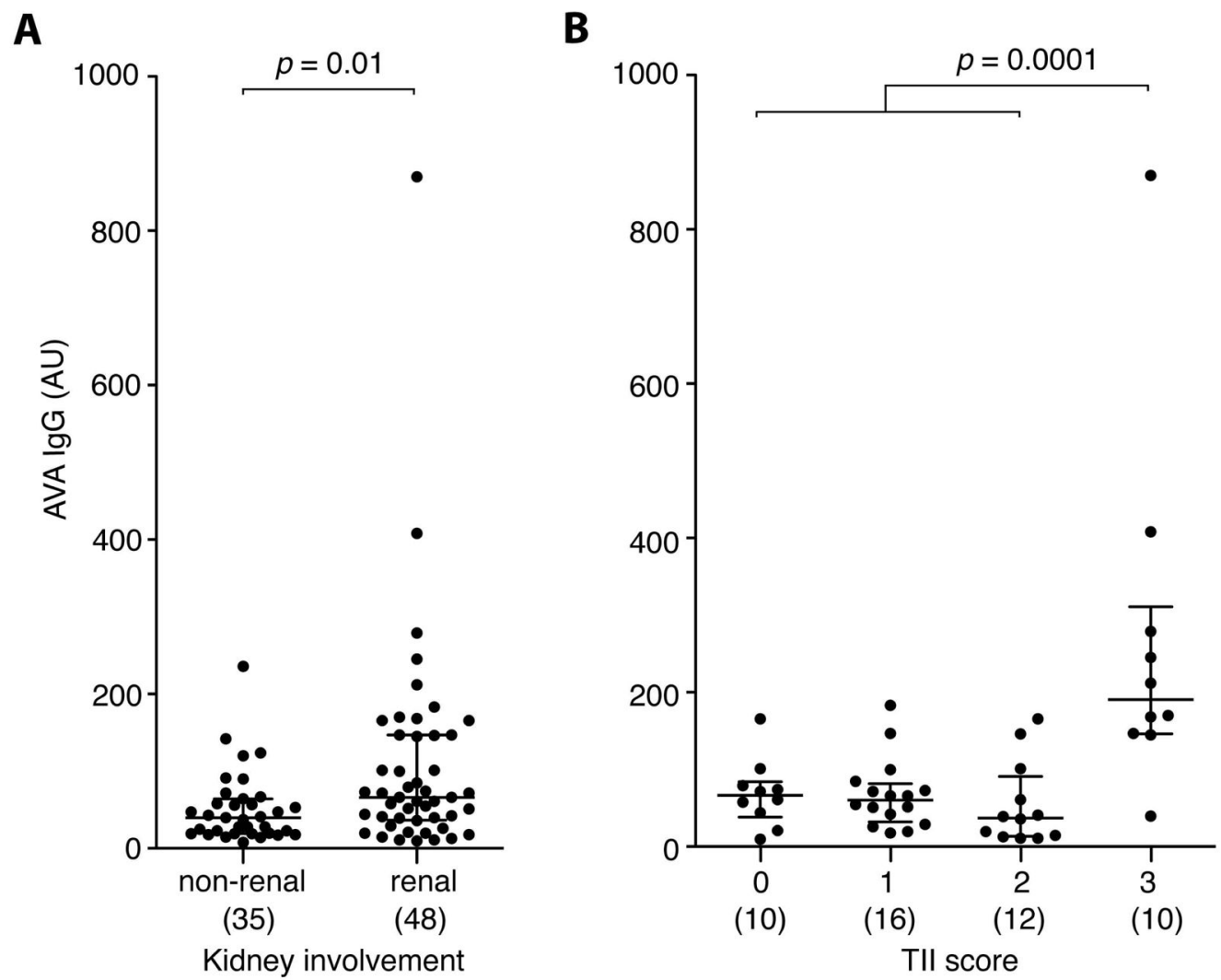

Figure 6.

Association of nephritis with anti-vimentin IgG levels in the serum of lupus patients. Individual serum samples from patients with SLE were used to probe vimentin arrays for $\mathrm{IgG}$ reactivity. Data-points represent individual serum samples. A, Patients were first grouped as having history of nephritis or not. B, Patients with nephritis were further characterized by degree of TII ( 0 is none, 1 is mild, 2 moderate and 3 severe). Titers are given in arbitrary units (AU), extrapolated from a serially diluted high AVA titered TII patient serum sample. Shown are p-values of two-tailed Mann-Whitney tests for indicated comparisons. Medians and interquartile ranges are given. For renal patients, the median time difference between biopsy and blood draw was 1.36 years (interquartile range $0.08-3.14$ years). The mean age at the time of serum draw was $33.1 \pm 9.7$ years. For the non-renal patients, average age at serum draw was $41.6 \pm 10.5$ years (vs. renal patients, $p<0.01]$ ). 